\title{
Ocean Ambient Noise and Its Directionality Measurement
}

\author{
SivaKumar.V.G', Dr.V.Rajendran ${ }^{2}$ Sailaja.M ${ }^{3}$ \\ ${ }^{1}$ (Assistant professor ECE, Sathyabama University, India) \\ ${ }^{2}$ (Professor, Physics/Ece, SSN College of Engineering) \\ ${ }_{3}^{3}(P G$ student, ECE, Sathyabama University, India)
}

\begin{abstract}
Sound is the effective way of communication underwater. It is the only radiation that can travel long ranges in water. Source, medium, detector (receiver) are three main elements for propagation. Ambient noise is the surrounding noise that is combined by different sources which impacts underwater sound communication and needs to be classified. Directionality attribute of the ambient noise are analyzed with respect to varying wind speeds, varying hydrophone depths and also for different frequencies. The ambient noise data are collected from the hydrophones placed at specific locations at Arabian Sea and Bay of Bengal. Low frequency noise arrives from the horizontal, as frequency increases, the vertical and near vertical contributions increase more rapidly than horizontal contributions. So vertical directionality is the key attribute of ambient noise to identify its source. In shallow water ambient noise and its vertical directionality are studied using vertical array of hydrophones. The properties of seabed vary spatially and thus provide an idea of sitespecific characteristics. The channel and it boundaries specification are fetched by the noise propagating through the shallow water along its path of transmission. Hence the environment information can be detected from the noise properties.
\end{abstract}

Keywords - Ambient noise, vertical directionality, hydrophone depths.

\section{INTRODUCTION}

Ambient noise is the background sounds which are spotted in the ocean, are complex. It is the combination of contributions of many different sources each differing in behavior and temporal and spatial dependence. Although there are many sources, the ambient noise itself can be expressed in terms of relatively small number of components, each produced by the contributions of a particular type of source. The effective way of characterizing the noise is to characterize the behavior of the individual components, each showing temporal and spatial variation related to behavior of the sources. The main components of ambient noise (a) sea surface noise or wind dependent noise (b) biological noise (c) traffic noise [1]. Wind dependent noise is the noise of wind and wave action at surface. The dependence of noise on wind speed is vital, since it provides a simple way of predicting the noise from weather forecasts, and if wind speed is measured together with measurements of ambient noise, this component can be separated from others. Biological noise forms a major component of ambient noise Biological choruses that result when large numbers of animals are calling commonly increase noise levels by $20 \mathrm{~dB}$ or more over typical background noise at low wind speeds and may extend over significant. Noise from fish, whales, and invertebrates are common.

Traffic noise is the background noise from the ships in an ocean and if the ship is too close, the noise produced will not be included. Ships at long distances can contribute to traffic noise if the propagation of sound is good, and more number of ships in ocean produce high traffic noise levels. Ambient noise in the real ocean is not usually uniform in azimuth, due to the presence of localized acoustic sources such as surface shipping, storm systems, ice cover, and underwater seismic events. The sources of noise in the ocean are (i) geophysical seismic waves, rain, hail and snow, hydrostatic and hydrodynamic sources such as bubbles, waves and wind turbulence (ii) human made - ship traffic, coastal of off shore activity, aircraft flying over the sea. (iii) biological sources - marine species (iv) thermal noise - due to bombardment of molecules.

The spectral classification of ambient noise: (i) Ultra-Low Band - The frequency band is below 1Hz.Examples for this band include the tides with lunar and solar periods and their harmonics, pressure due to wave. (ii)Infrasonic band - The frequency range is between 1 to $20 \mathrm{~Hz}$. The ship noise begins to be strong in the frequency region of 5 to $20 \mathrm{~Hz}$. In shallow water where the shipping noise won't be appreciable, the spectrum will fall off and depends on wind speed for the entire band. This band contains the strong blade-rate fundamental frequency of propeller-driven vessels, one or two of its harmonics, and the band is therefore of subject to low frequency passive SONARS. (iii)Low sonic band (20 to $200 \mathrm{~Hz})$ - This frequency band is characterized by the noise of distant shipping in areas and man made activities other than shipping. Away from areas of shipping the band depends on wind speed as in case of lower and higher frequencies. Below $20 \mathrm{~Hz}$ bands and above $200 \mathrm{~Hz}$ the noise is dependent on wind speed. In the band 20 to $200 \mathrm{~Hz}$, the non-wind 
dependent noise, with peaks at 20 and $60 \mathrm{~Hz}$, is attributed to a combination of biological sources, shore activities $(60 \mathrm{~Hz})$ and distant ocean ship traffic. (iv)High sonic band (200 to $50 \mathrm{KHz})$ - If the noise level is related to wind speed, as is certain to be the case at kilohertz frequencies; it allows using a hydrophone as an anemometer for measurement of wind speed at remote underwater locations. This band includes noise due to wind and heavy rain. (v)Ultrasonic Band (> $50 \mathrm{kHz}$ ) - At frequencies from 50 to $200 \mathrm{kHz}$, depending on wind speed, thermal noise begins to dominate this band. Thermal noise is the noise of molecular bombardment. The noise levels to increase with decreasing latitude. Thus the noises prevailing under ocean are classified based on the frequency spectrum between various ranges. This classification enhances the study and research of under water acoustics.

\subsection{Basic Properties of Acoustic Waves}

The characterization of acoustic waves can be done by using amplitude, frequency, wavelength, phase, speed and intensity. The amplitude of sound wave and maximum distance a vibrating particle is displaced from rest are proportional with each other. Small variations in amplitude produce weak or quiet sounds, while large variations produce strong or loud sounds [2]. The rate of oscillation or vibration of the wave particles is referred as the frequency of a sound wave. To the human ear, the increase in frequency is observed as a high pitched sound and increase in amplitude is found to be similar to louder sound. The wavelength of a wave is the distance between two successive compressions or the distance the wave travels in one cycle of vibration.

The alignment of one propagating wave with another in accordance to time is called as the phase of acoustic waves. Complex sounds are obtained from simple sinusoidal waves. If the amplitude of waves is sum of the interfering waves then they are said to be in same phase and two waves with opposite phases will destructively interfere to cancel each other out.

\subsection{The propagation of underwater noise}

The basic parameters to describe the propagation of underwater noise are:

(1) Source: the noise source, example could be ship, sonar etc. Parameter of interest = source level (SL)

(2) Path or medium of transmission: the water column. Parameters of interest include transmission loss (TL), and ambient noise level (NL)

(3) Receiver: whale, hydrophone etc. Parameters of interest include signal to noise ratio (SNR), received sound intensity level (RL) and detection threshold (DT).

Sonar Equation is:

$$
\mathrm{RL}=\mathrm{SL}-\mathrm{TL}(1)
$$

Where RL is the received level, SL is the source level and TL is the transmission loss. Transmission loss is due decrease in intensity of a sound as it propagates via a medium, and this loss is because of spreading, absorption, scattering, reflection and rarefaction. By adding the effects of geometrical spreading, absorption and the transmission loss anomaly (A), transmission loss TL can also be determined. The transmission loss anomaly includes scattering loss and losses due to reflection and rarefaction at boundary interfaces.

\subsection{Noise Variability in Shallow Water}

Shallow water is noteworthy for its variability. Rapid noise changes can be recognized in waters close to shore and in busy harbors are dynamic locations. Half the noise comes from an area on the surface of radius equal to the hydrophone depth, and the intensity is independent of measurement depth in the absence of attenuation and bottom effects. The intensity of wind noise increases as the square of the wind speed. In shallow water, propagation sometimes affects the ambient background, though in a different way than it does distant shipping noise in deep water [3].Shallow water ambient noise in the midfrequency band consists of wind-driven component with shipping and biological components.

Theoretical models of surface-generated noise in a shallow ocean indicate that the vertical directionality is strongly controlled by bottom interface and the directional responses are also dependent on the source directionality parameter and sound-speed profiles [4]. The detection and localization using a vertical array in shallow water can be improved if the noise field is directional and the signal arrives in the null of the noise field. Most of the studies on shallow-water noise directionality have taken place in water depths of above $100 \mathrm{~m}$, and not carried out in very shallow waters. Measurements of directionality using vertical line array sonobuoys have been used for determining seabed critical angle [5], and potential geoacoustic parameters have also been inferred by inversion of noise using reflection loss since vertical directionality is strongly influenced by seabed reflections [6]. 


\section{Vertical Directionality}

A mbient noise environments can be characterized by the directionality of noise in addition to the pressure spectral density. The direction of noise can be determined by processing multiple channels of a hydrophone array. Directional behavior is consistent with the view that low-frequency noise originates at great distances and arrives at the measurement hydrophone principally via horizontal paths, suffering little attenuation. Vertical directionality of the shallow-water noise field is determined primarily by the geoacoustic properties of the seabed rather than by any temporal variations in source distributions. Smooth spectral variations in the vertical directionality of the noise field are also characteristic of low-shipping areas.

\subsection{Variation of Vertical Directionality with Depth}

Because of different propagation to different depths, the vertical directionality of noise traveling within the Deep channel varies with depth. On the axis of the channel in deep water of uniform depth, a ray diagram shows that sound from distant surface sources can arrive only at angles of +12 to $15^{\circ}$ to the horizontal; at smaller angles only low-level diffracted, scattered or slope-enhanced energy can be received[7]. Ship noise originating in the dense traffic in the major shipping lanes toward the north travels down the channel along paths near the channel axis to the measurement location .Ship noise originating in the dense traffic in the major shipping lanes toward the north travels down the channel along paths near the channel axis to the measurement location. Noise process at shallow waters is highly variable due to high variability of ship traffic, wave guide nature, reflection of noise from the bottom and surface, biological activities. Variations of ship traffic and of wind speed over a period of time causes variation of noise level and in the absence of shipping wind dominate the entire frequency range.

\subsection{Power Spectrum Estimation}

A spectrum is a condition that is not limited to a specific set of values but can vary infinitely within a continuum. The word saw its first scientific use within the field of optics to describe the rainbow of colors in visible light when separated using a prism; it has since been applied by analogy to many fields other than optics.

\subsubsection{Wiener-Khintchine Theorem}

Let $\mathrm{x}(\mathrm{n})$ be a WSS random process with autocorrelation sequence:

$$
r_{x x}(m)=E\left[x(n+m) x^{*}(n)\right]
$$

The power spectral density is defined as the Discrete Time Fourier Transform of the autocorrelation sequence:

$$
p_{x x}(f)=T \sum_{n=-\infty}^{\infty} r_{x x}(m) e^{-i 2 \pi f m T}
$$

where $T$ is the sampling interval. The signal is assumed to be band limited in frequency to $\pm 1 / 2 \mathrm{~T}$ and is periodic in frequency with period $1 / \mathrm{T}$.

The inverse DTFT is:

$$
r_{x x}(m)=\int_{-1 / 2 T}^{1 / 2 T} P_{x x}(f) e^{i 2 \pi f m T} d f
$$

And, $r_{x x}(m)$ is the average power. Due to the property $r_{x x}(m)=r_{x x}(m)$,the PSD must be a strictly real, nonnegative function. If a process is ergodic then ensemble averages can be replaced with "time" averages. Power spectrum definition using time averages:

$$
P x x(f)=\lim m \longrightarrow \infty E\left\{\frac{1}{(2 M+1) T}\left|t \sum_{n=-M}^{M} x(n) e^{-i 2 \pi f n T}\right|^{2}\right\}
$$

This is the basis of the periodogram method for estimating the PSD. The Fourier transform of $x(n)$ is computed, which can be computed via the FFT. To reduce the variance in the estimate, many spectra can be averaged.

Steps In Spectral Analysis:

- Convert the voltage output of hydrophone to corresponding pressure value.

- Filter the wide band data to limit to the band of interest.

- Take FFT of the filtered data and convert to dB scale. 
- Analyze the data for various observations.

Parameters for analysis

- Sampling rate and duration of sampling.

- Filter type and bandwidth of the filter.

- FFT window size and the type of window.

- Overlapping length.

\subsubsection{Cross Power Spectral Density}

The DTFT (discrete time Fourier Transform) of the cross-correlation is called the cross-power spectral density, or "cross-spectral density," "cross-power spectrum". It is the distribution of power per unit frequency. From CPSD, angle of incidence can be obtained, using which directionality can be found. The cross power spectral density Pxy of the discrete-time signals $\mathrm{x}$ and $\mathrm{y}$ using the Welch's averaged, modified periodogram method of spectral estimation. The cross power spectral density is the distribution of power per unit frequency and is defined as:

$$
\operatorname{Pxy}(\omega)=\sum_{m=-\infty}^{\infty} R_{x y}(m) e^{-j \omega m}
$$

The cross-correlation sequence is defined as:

$$
R x y(m)=E\left\{x_{n+m} y * n\right\}=E\left\{x_{n} y *_{n-m}\right\}
$$

where $x_{\mathrm{n}}$ and $y_{\mathrm{n}}$ are jointly stationary random processes, and $E\{\cdot\}$ is the expected value operator. For real $\mathrm{x}$ and $\mathrm{y}$, cpsd returns a one-sided CPSD and for complex $\mathrm{x}$ or $\mathrm{y}$, it returns a two-sided CPSD.

CPSD uses the default values as mentioned below

(i) $\mathrm{Nfft}$

FFT length which determines the frequencies at which the PSD is estimated. For real $\mathrm{x}$ and $\mathrm{y}$, the length of Pxy is (nfft/2+1) if nfft is even or (nfft+1)/2 if nfft is odd. For complex x or y, the length of Pxy is nfft. If $\mathrm{nfft}$ is greater than the signal length, the data is zero-padded. If nfft is less than the signal length, the segment is wrapped using data wrap so that the length is equal to nfft. Maximum of 256 or the next power of 2 greater than the length of each section of $\mathrm{x}$ or $\mathrm{y}$.

(ii) Sampling Frequency $\left(\mathrm{f}_{\mathrm{s}}\right)$

The rate at which the data are sampled.

(iii) Window

Windowing function and number of samples to use for each section. Periodic Hamming window of length to obtain eight equal sections of $\mathrm{x}$ and $\mathrm{y}$.

(iv) noverlap

Number of samples by which the sections overlap. Value to obtain 50\% overlap.

\section{Ambient noise buoy}

Ambient noise data buoy is a system used for continuous measurement of under water noise. It is integrated with the following instruments:

1. Hydrophones

2. Autonomous data acquisition system 


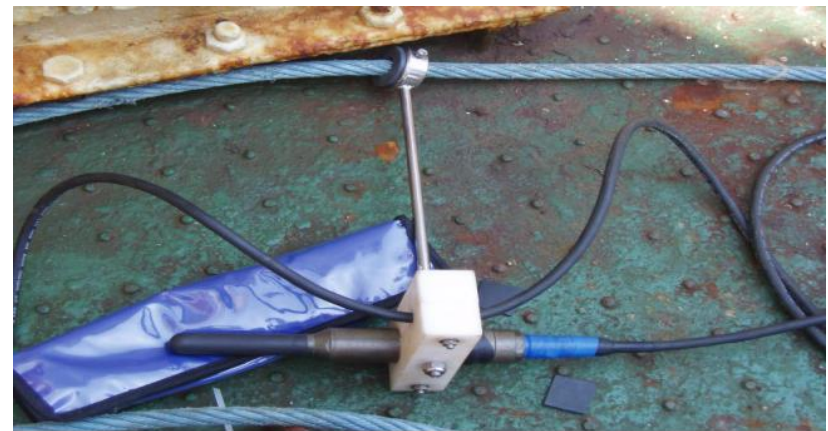

Fig 1: Hydrophone with fixture

Ambient noise buoy consist of

- Wind sensor - The Anemometer of model is incorporated with the ambient noise Data Buoy.

- DGPS is an integrated GPS/Beacon receiver with built-in antennas that provide differential corrected positioning data in for navigation requiring high degree accuracy.

- Acoustic release - One end of the acoustic release integrated with the Data buoy moorings and another end connected to the combination rope, which was connected to anchor weight of the data buoy.

- Mooring System

Due to the vulnerability of surface based noise measurement system, a subsurface automated system was designed and developed.

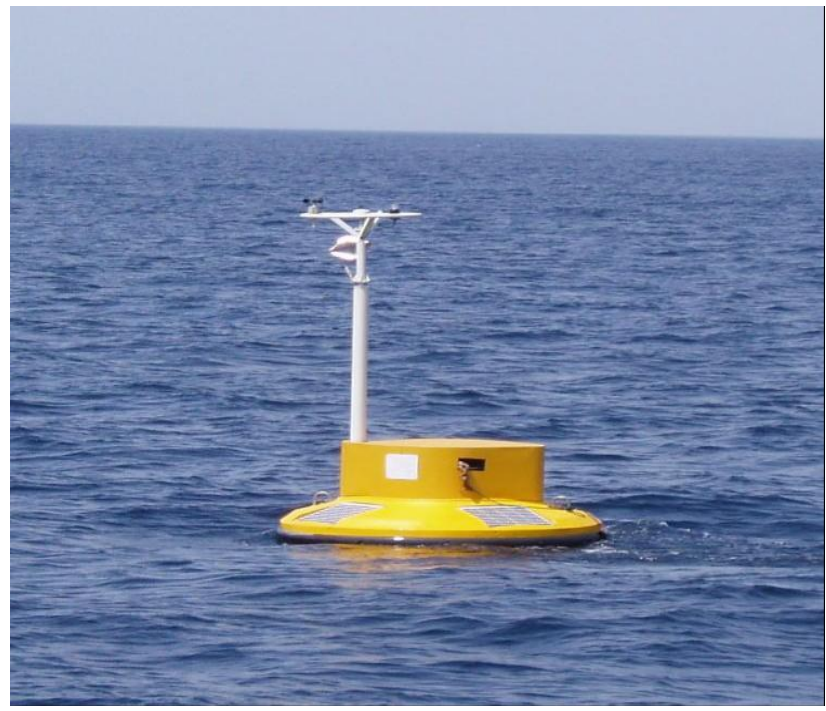

Fig 2: Ambient Noise Buoy

\section{Formula Used}

The angle of incidence for the signal received by the hydrophone is given by:

$\theta_{k l}(f)=\cos ^{-1}\left[\frac{P D_{k l}}{d}\right]=\cos ^{-1}\left[\frac{P \phi_{k l}(f)}{2 \pi f d}\right] \quad$ Eq (1)

$P$ is Propagation velocity of source signal

$D_{k l}$ is time delay

$\mathrm{d}$ is separation between the receivers

$\phi_{k l}(f)$ is phase shift in radians

For non-dispersive propagation $\mathrm{P}$ is constant, phase shift is linear, whereas for dispersive propagation, $\mathrm{P}$ varies with frequency phase shift is nonlinear. If the source is sufficiently distant to be approximated by a plane wave at the location, the time delay can be interpreted as an angle of incidence [8]

For non-dispersive propagation: 


$$
\theta_{k l}(f)=\cos ^{-1}\left[\frac{P b_{k l}}{2 \pi d}\right]
$$

Where, $b_{k l}=\frac{\phi_{k l}(f)}{f}, b_{k l}$ is slope of phase curves in radians per Hz.

Ambient sea noise directionality is determined from the output of an array of receiving hydrophones. To obtain the ambient sea noise directionality, the cross spectral matrix between the array hydrophone outputs is made use off. Array used here is an equally spaced five-element hydrophone array, with an aperture of $0.6 \mathrm{~m}$.

\section{Directionality for Various Parameters}

The study of vertical directionality is carried out with regard to various wind speed, frequency, depths. The graph is plotted for all the above mentioned constraint and the result is analyzed.

\subsection{Directionality for various Wind Speed}

For different wind speed of $3.62 \mathrm{~m} / \mathrm{s}$ and $4.21 \mathrm{~m} / \mathrm{s}$ at depth of $12 \mathrm{~m}$, frequency of $2000 \mathrm{~Hz}$, the graph is plotted as

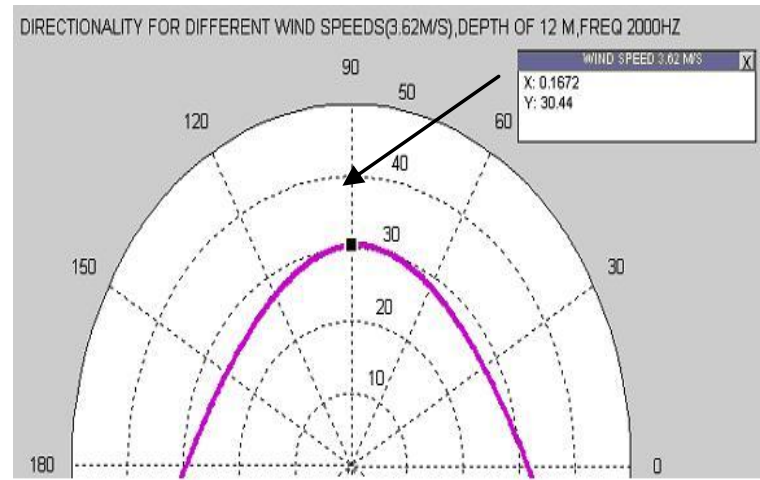

Fig 3: Directionality for wind speed of $3.62 \mathrm{~m} / \mathrm{s}$

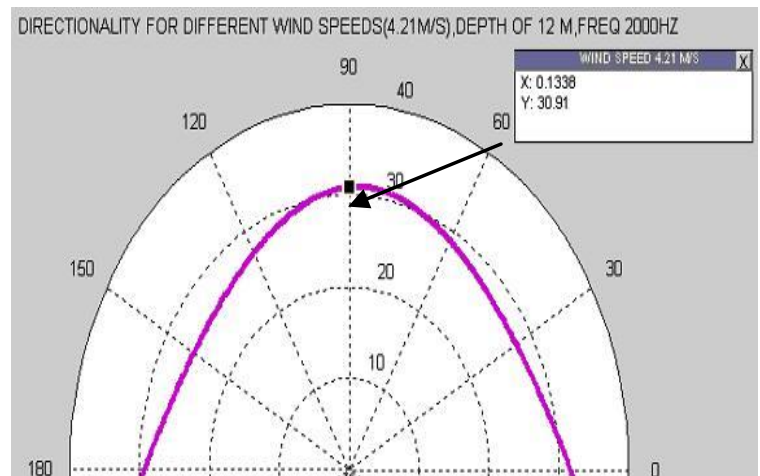

Fig 4: Directionality for wind speed of $4.21 \mathrm{~m} / \mathrm{s}$

As the wind speed increases from $3.62 \mathrm{~m} / \mathrm{s}$ to $4.21 \mathrm{~m} / \mathrm{s}$, the noise level also shows slightly varies from its amplitude from 30.44 to 30.91 for the frequency of $2000 \mathrm{~Hz}$ It is indicate that for the particular direction the noise level increases for the increase of wind speed.

\subsection{Directionality for Different Frequencies}

For different frequencies of signal $3000 \mathrm{~Hz}, 4000 \mathrm{~Hz}$, with the wind speed of $3.62 \mathrm{~m} / \mathrm{s}$ at depth of $12 \mathrm{~m}$, the graph is plotted.

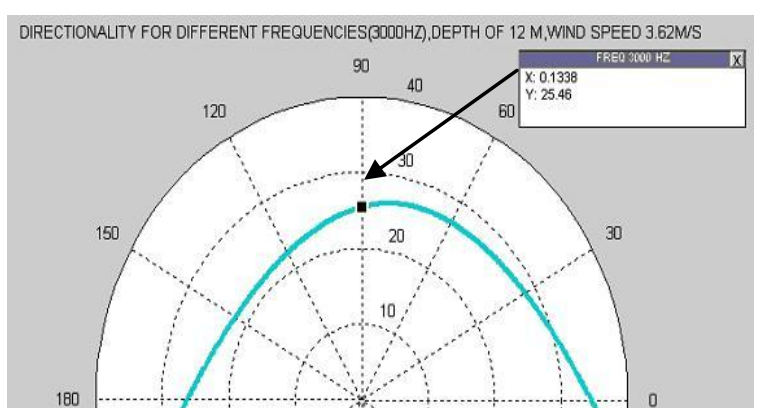

Fig 5: Directionality for frequency of $3000 \mathrm{~Hz}$

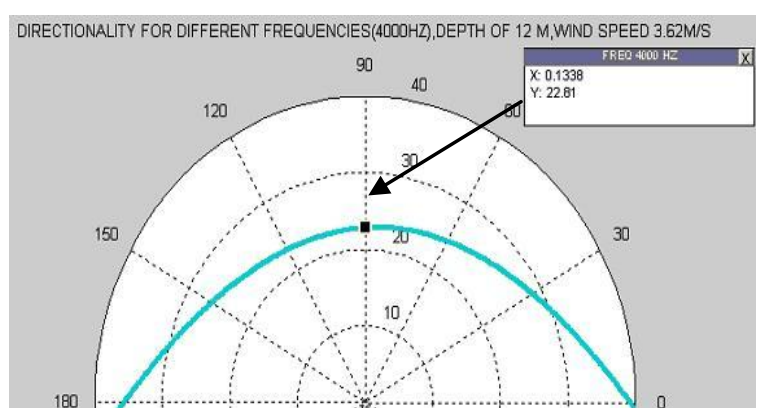

Fig 6: Directionality for frequency of $4000 \mathrm{~Hz}$

As the frequency increase, the level of noise amplitude decreases. More noise arrives from near horizontal direction, which can be characteristic of low frequency ship noise dominating surface noise. As the frequency increases the horizontal dominance decreases. At $3000 \mathrm{~Hz} \& 4000 \mathrm{~Hz}$, the noise is predominantly from the vertical contributions. The amplitude values are 25.46 for $3000 \mathrm{~Hz}, 22.81$ for $4000 \mathrm{~Hz}$. 


\subsection{Directionality for Different Depths}

For different depths of $12 \& 15 \mathrm{~m}$ and at a frequency of $2000 \mathrm{~Hz}$, the graph is plotted as

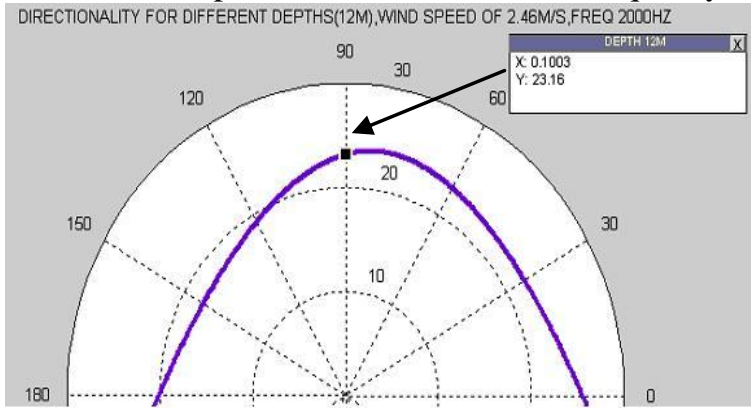

Fig 8: Directionality for depth of $12 \mathrm{~m}$

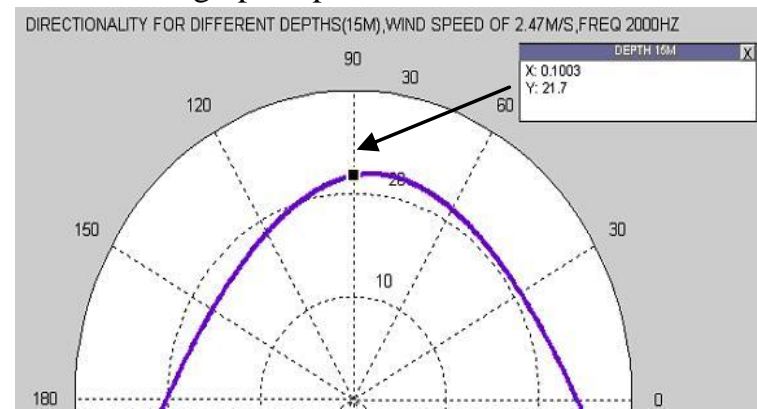

Fig 9: Directionality for depth of $15 \mathrm{~m}$

For depth change from $12 \mathrm{~m}$ to $15 \mathrm{~m}$, there is decrease in the noise level as shown. For $2000 \mathrm{~Hz}$ signal the noise levels varies with depth, the variation of noise level is due to effects of scattering and absorption. The value of amplitude changes from 23.16 to 21.7 for the depth change of $12 \mathrm{~m}$ to $15 \mathrm{~m}$.

Because of the time variability of sources of noise, ambient noise varies for a given location. The directional distribution shown here gives the properties of the noise field independently of the geometry of the particular array used to measure it. At near the horizontal, noise emanating from distant sources arrives at the array, whose high frequency components are attenuated strongly in transit. Noise incident from the vertical is subject to less attenuation, because of much shorter path lengths involved. With increasing frequency, surface noise relative to shipping noise.

\section{Conclusion}

With help of directionality concept, the direction from which the ambient noise received by the hydrophones can be found. This gives how the noise level depends on variation with wind speeds, frequencies and depths for particular direction. The time variability of sources of noise, is because of ambient noise varies for a given location. The directional distribution shown here gives the properties of the noise field independently of the geometry of the particular array used to measure it.

Using the concept of directionality the further progress can be done in the field of under water acoustics. It is to detect classify and localize the objects, navigation, obstacle avoidance. Other major applications would be Acoustic transmission and reception of voice or data signals. Future work can be done on resource availability, monitoring marine environment. For various depths of ocean, the directionality parameter can be studied and further processing of data can be done to predict the available resources under ocean and future work can be carried out to enhance the study of underwater acoustics.

\section{References:}

[1] Douglas H. Cato, Ocean Ambient Noise: Its Measurement and Its Significance to Marine Animals, Defence Science and Technology Organisation, and University of Sydney Institute of Marine Science, Sydney, NSW 2006 Australia, Proceedings of the Institute of Acoustics.

[2] Editors: Mark Simmonds, Sarah Dolman and Lindy Weilgart, Oceans of Noise 2004, A WDCS Science Report, WDCS, the Whale and Dolphin Conservation Society, Brookfield House, 38 St Paul St, Chippenham, Wiltshire, SN15 1LJ, (chapter 2.1.1).

[3] R. J. URICK, Adjunct Professor, The Catholic University of America Washington, D.C. 20046 ,Published by undersea warfare published by Undersea warfare technology office naval sea systems command department of the navy Washington,D.C.20362.

[4] M. C. Sanjana and G. Latha, Member, IEEE, Peer-Reviewed Technical Communication, Midfrequency Ambient Noise Notch using Time-Series, Measurements in Bay of Bengal, IEEE Journal of Oceanic Engineering, VOL. 37, NO. 2, APRIL 2012255.

[5] M. J. Buckingham and S. A. S. Jones, A new shallow ocean technique for determining the critical angle of the seabed from the vertical directionality of the ambient noise in the water column, J. Acoust. Soc. Amer., vol. 81, no. 4, pp. 938-946, 1987.

[6] C. H. Harrison and D. G. Simons, Geoacoustic inversion of ambient noise: A simple method, J. Acoust. Soc. Amer., vol. 112, no. 4, pp. 1377-1389, 2002.

[7], [8], (8) Allan G.Piersol, Time Delay Estimation Using Phase Data, IEEE Transactions on Acoustics, speech and signal processing. 